\title{
Simplified phenotypic tests for identification of Acinetobacter spp. and their antimicrobial susceptibility status
}

\author{
K. PRASHANTH and S. BADRINATH
}

Department of Microbiology, Jawaharlal Institute of Postgraduate Medical Education and Research (JIPMER), Pondicherry, India

\begin{abstract}
Acinetobacter spp. have been found to be responsible for an increasing number of nosocomial infections. During a 16-month period, 22 patients hospitalised mainly in the respiratory intensive care unit (RICU), paediatric and other medical wards were investigated either for infection or colonisation by Acinetobacter spp. Of the 45 isolates of Acinetobacter detected among the total of 425 non-fermenters encountered, 24 representative isolates were selected for extended phenotypic identification. Four environmental isolates were also included in the study. These 28 isolates were typed by biotyping and antibiotyping, which helped in delineating the Acinetobacter spp. into 12 phenotypes and two distinct antibiotypes respectively. A sudden increase of cases of acinetobacter infection suggested that three outbreaks during the study period were due to phenotypes 1 and 2 of $A$. calcoaceticus- $A$. baumannii complex (Acb). Strains of $A c b$ complex showed multiple drug resistance and were sensitive only to netilmicin. A comparatively high proportion of resistance to amikacin $(48 \%)$ was also detected among these strains by the agar dilution method. The RICU environment was recognised as an important reservoir for the resistant outbreak strain $(A c b-1)$ which was probably leading to persistent colonisation and recurrent infections.
\end{abstract}

\section{Introduction}

Acinetobacter spp. have been implicated in recent years as important nosocomial pathogens, especially in intensive care settings [1-3]. Despite their low pathogenic potential they are being reported increasingly as the causal organism of numerous hospital outbreaks in several countries [4-6]. In a recent international multicentre study, Acinetobacter spp. were ranked amongst the 10 organisms most commonly causing septicaemia in 18 of 44 large European hospitals [7]. However, community-acquired pneumonia due to Acinetobacter species is rare, as evidenced by the paucity of reports $[8,9]$.

Despite the increasing significance and frequency of multiresistant acinetobacter infections, many clinicians and microbiologists still lack an appreciation of the importance of these organisms in hospitals, in part

Received 4 Feb. 1999; revised version accepted 20 Dec. 1999.

Corresponding author: Dr S. Badrinath. because of their confused taxonomic status [9]. In India, very few studies of Acinetobacter spp. have been reported and, in view of their increasing importance in nosocomial infections, further study is warranted in this part of the world $[10,11]$.

In the present study an attempt was made to type the Acinetobacter isolates obtained from various sources by a simplified phenotypic identification scheme with a numerical approach [12-14] and also to determine their antimicrobial susceptibility.

\section{Material and methods}

Bacterial strains

Forty-five isolates of Acinetobacter spp. were included in the study. These had been isolated mainly from blood, tracheal aspirate, pus and other body fluids over a period of 16 months (Oct. 1996-Feb. 1998), mainly from patients admitted to the respiratory intensive care unit (RICU; six beds) (34 patients), paediatric ward (5) and other medical wards (6) in the 700-bed JIPMER hospital. The major reasons for admission to the RICU are mechanical ventilation for respiratory failure, 
postoperative critical care and organ support following multiple trauma. Such admissions are usually for 2-4 days. Four Acinetobacter isolates were from 10 environmental samples (hands of attending clinicians, ventilator monitors, humidifier and endotracheal tube surfaces) obtained during the period of high incidence of isolation of this bacterium from clinical specimens from patients in the RICU. Environmental samples were collected according to the method of Seifert et al. [15]. The reference strains were included in the study. All isolates were preserved in glycerol broth at $-70^{\circ} \mathrm{C}$ until tested.

\section{Phenotypic identification}

All isolates were subjected to simplified phenotypic tests as described by Gerner-Smidt et al. $[12,14]$ (Table 1). Briefly, tests for growth at $37^{\circ} \mathrm{C}, 30^{\circ} \mathrm{C}$ and $44^{\circ} \mathrm{C}$ were performed in brain heart infusion broth in a water bath. Sheep blood (5\%) agar plates were used to detect haemolysis. Glucose oxidation was determined in Hugh and Leifson's medium containing glucose 1\% and the gelatin stab method was used to test for gelatin liquefaction [16]. The assimilation tests were performed in a liquid medium containing a mineral base as described by Stanier et al. [17] with the addition of an appropriate carbon source in a $0.1 \% \mathrm{w} / \mathrm{v}$ concentration. t-Aconitate, L-histidine, malonate, histamine and citrate were used for assimilation tests. Additional carbon source assimilation tests such as DL-4 aminobutyrate and L-phenylalanine were used for haemolytic isolates (DNA groups 4,6 and 14). Susceptibility to penicillin $\mathrm{G}(10 \mu \mathrm{g})$ and chloramphenicol $(30 \mu \mathrm{g})$ was tested by the disk diffusion method, as they were used as phenotypic tests along with the rest [13]. The susceptibility tests were read after overnight incubation, and the tests for haemolysis and growth at $37^{\circ} \mathrm{C}$ and $44^{\circ} \mathrm{C}$ were read on the first and second day; the assimilation tests were read after incubation for 1,2, and 6 days. All were incubated at $30^{\circ} \mathrm{C}$, except for the tests for growth at $37^{\circ} \mathrm{C}$ and $44^{\circ} \mathrm{C}$, and for gelatin liquification the incubation temperture was $22^{\circ} \mathrm{C}$.

\section{Antimicrobial susceptibility}

Antimicrobial susceptibility was determined by both the Kirby-Bauer disk diffusion method [18] and an agar dilution method for minimum inhibitory concentration (MIC) determination [19]. Escherichia coli ATCC 25922 was included as control. The tests were read after overnight incubation and the following antibiotics were used: cefotaxime, ceftazidime, amikacin, gentamicin, ampicillin, piperacillin, netilmicin, ciprofloxacin, norfloxacin, cefazolin and cephalexin. Only the first three antibiotics were used for MIC detection. Susceptibility results were analysed with the WHONET4 programme.

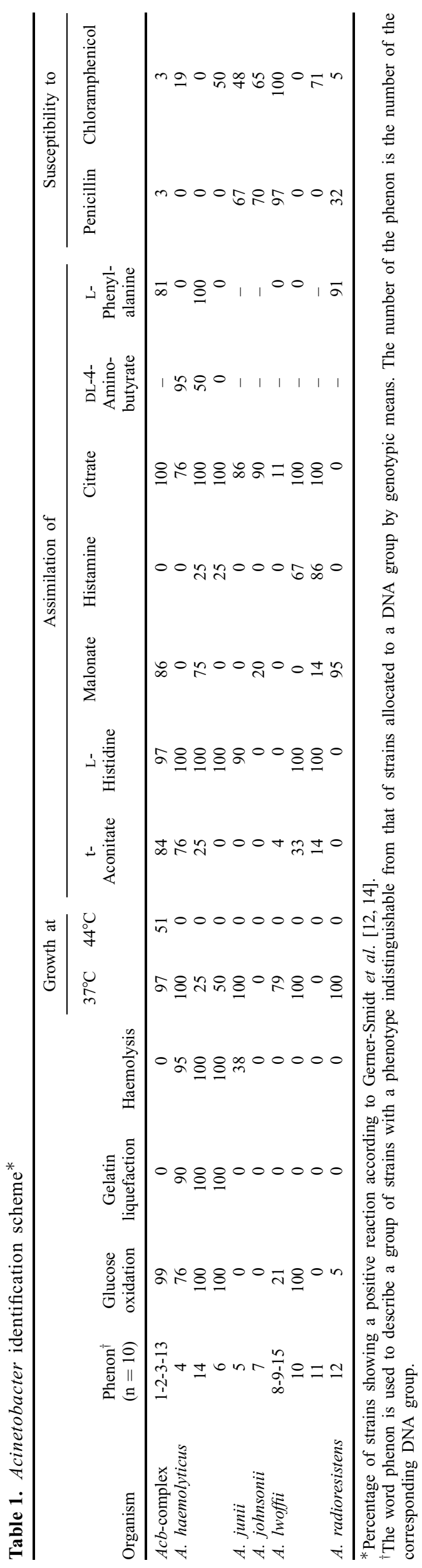




\section{Results}

In all, 45 isolates were obtained during the study period of 16 months. Of these, 34 isolates were from multiple sites in 11 patients in the RICU (Table 2). In eight cases, apart from tracheal aspirate, Acinetobacter spp. were repeatedly isolated from blood. In cases where multiple isolates were obtained from the same patient, after performing preliminary biochemical testing and antibiotyping a representative strain(s) was subjected to extended phenotypic characterisation. In two cases, two different strains were isolated from each patient. Hence the study population consisted of 28 strains, which included both clinical (ICU, 13 strains; medical ward, 6; paediatric ward, 5) and environmental isolates (4) (Table 3).

Of the 28 isolates, 22 were biovar anitratus and 6 were biovar lwoffii. With the simplified phenotypic identification scheme, the 28 strains tested belonged to only 12 phenotypes (Table 4). The first five phenotypes (20 strains) were identified as members of the $A$. calcoaceticus-A. baumannii complex (Acb-complex; phenotype 1-5, phenon 1-2-3-13). One strain was attributed to the $A c b$-complex (phenotype 6) despite failing to acidify glucose. One strain was identified as A. haemolyticus (phenotype 7) and another as A. lwoffii (phenotype 8). The remaining four phenotypes $(9,10$, 11 and 12) did not fit the phenotypic description of any single phenon. Phenotype 9 was like the typical $A$. haemolyticus phenotype, except for assimilation of malonate ( $A$. haemolyticus-like). Phenotype 10 showed the typical $A$. lwoffii phenotype (A. lwoffii-like) except for assimilation of L-histidine. Phenotype 11, which comprised two strains (phenon 11-like), resembled phenon-11 in every respect except in failing to grow at $37^{\circ} \mathrm{C}$. Phenotype 12 resembled the members of phenon $8-9-15$, as it grew at $37^{\circ} \mathrm{C}$ and was susceptible to chloramphenicol.
Table 3. Environmental isolates of Acinetobacter spp.

\begin{tabular}{|c|c|c|c|}
\hline $\begin{array}{l}\text { Sample } \\
\text { no. }\end{array}$ & $\begin{array}{l}\text { Date of } \\
\text { isolation }\end{array}$ & Site & $\begin{array}{l}\text { Organism } \\
\text { isolated }\end{array}$ \\
\hline 1 & $12-10-96$ & Ventilator monitor & None \\
\hline 2 & $20-10-96$ & Humidifier & None \\
\hline 3 & $27-09-97$ & $\begin{array}{l}\text { Endotracheal tube surface, } \\
\text { humidifier }\end{array}$ & $A c b-1$ \\
\hline 4 & $10-10-97$ & Humidifier & $A c b-4$ \\
\hline 5 & $23-02-98$ & Humidifier & A. lwoffi-like \\
\hline 6 & $\ldots$ & Forearm & None \\
\hline 7 & $\ldots$ & Monitoring surfaces & None \\
\hline 8 & $26-02-98$ & Forefinger & $A c b-1$ \\
\hline 9 & $\ldots$ & Axilla & None \\
\hline 10 & $\ldots$ & Bedsides & None \\
\hline
\end{tabular}

\section{Antimicrobial susceptibility testing}

The MIC results for 28 Acinetobacter isolates are shown in Table 5. It also shows the range of the MIC observed, as well as the MIC50, MIC90 and percentage of resistant strains among $A c b$ (21) and non- $A c b$ (7) isolates. A slightly higher percentage of resistance to amikacin (48\%) was noted among strains of the $A c b$ complex when compared with that of the other two antibiotics tested (43\%). Many strains (14) showed high-level resistance to amikacin $(\geqslant 256 \mathrm{mg} / \mathrm{L})$. The MIC90 of cefotaxime was $\geqslant 256 \mathrm{mg} / \mathrm{L}$ for 10 strains, while that of ceftazidime was $128 \mathrm{mg} / \mathrm{L}$ for eight strains. The disk diffusion method showed cefazolin, piperacillin, gentamicin and norfloxacin as having very little activity, with the percentage of resistance ranging from 68 to $96 \%$. Almost all strains were resistant to cefazolin and cephalexin. Generally, strains of the $A c b$ complex were more resistant to all the antibiotics tested than strains belonging other phenons.

The agar dilution technique showed that the overall percentage of strains susceptible to cefotaxime and ceftazidime was $50 \%$ and $58 \%$ respectively. The overall susceptibility for amikacin was similar to that of

Table 2. Respiratory intensive care patients positive for Acinetobacter spp.

\begin{tabular}{|c|c|c|c|c|c|c|}
\hline $\begin{array}{l}\text { Patient } \\
\text { no. }\end{array}$ & Age/sex & Diagnosis & Outcome & Sites positive & $\begin{array}{l}\text { Date of first } \\
\text { isolation }\end{array}$ & $\begin{array}{l}\text { Phenotype } \\
\text { isolated }\end{array}$ \\
\hline 1 & $40 / \mathrm{F}$ & $\begin{array}{l}\text { Post-surgery (oesophagostomy) } \\
\text { septicaemia }\end{array}$ & Discharged & $\begin{array}{l}\text { Blood*, PF } \\
\text { sputum, TA, pus }\end{array}$ & $10-10-96$ & $A c b-2$ \\
\hline 2 & $30 / \mathrm{M}$ & Intracranial space-occupying lesion & Died & Blood* & $18-10-96$ & $A c b-2$ \\
\hline 3 & $48 / \mathrm{F}$ & Strangulated hernia, septicaemia & Died & Blood $^{* \dagger}$, TA & $26-10-96$ & $A c b-6$ \\
\hline 4 & $20 / \mathrm{F}$ & Aspiration pneumonia & Died & Blood, TA & $26-10-96$ & $A c b-2$ \\
\hline 5 & $47 / \mathrm{M}$ & Peritonitis & Discharged & TA, blood & $28-02-97$ & $A c b-2$ \\
\hline 6 & $20 / \mathrm{F}$ & Cardiac arrest & Discharged & Blood, TA & $18-09-97$ & $A c b-3, A c b-2$ \\
\hline 7 & $11 / \mathrm{F}$ & Demyelinating disease, pneumonia & Transferred & $\begin{array}{l}\text { Blood }^{*}, \text { TA, } \\
\text { sputum }\end{array}$ & $13-09-97$ & $A c b-2$ \\
\hline 8 & $74 / \mathrm{M}$ & Multiple organ dysfunction & Died & TA, bile, blood* & $29-09-97$ & $A c b-1$ \\
\hline 9 & $63 / \mathrm{M}$ & Aspiration pneumonia & Discharged & Blood $^{* \dagger}$, TA & $23-02-98$ & $A c b-5, A c b-2$ \\
\hline 10 & $20 / \mathrm{M}$ & Septicaemia & Discharged & TA, blood* & $27-02-98$ & $A c b-1$ \\
\hline 11 & $45 / \mathrm{F}$ & Trauma, ARDS & Transferred & TA, blood* & $28-02-98$ & $A c b-2$ \\
\hline
\end{tabular}

TA, tracheal aspirate; PF, pleural fluid; ARDS, adult respiratory distress syndrome.

* Repeat isolation from blood.

${ }^{\dagger}$ Colonisation only, as there were no related clinical findings. 
ceftazidime $(58 \%)$ and only $3 \%$ were of intermediate susceptibility. Strains moderately sensitive (intermediate range) to cefotaxime (23\%) were more common than for other antibiotics tested. The disk diffusion method showed netilmicin and amikacin to have the greatest activity against the strains, with overall sensitivity of $64 \%$ and $48 \%$ respectively. The $A$. lwoffi, A. lwoffii-like, A. haemolyticus and A. haemolyticus-like strains were generally more susceptible to quinolones, piperacillin, gentamicin and amikacin than the $A c b$-complex strains.

\section{Discussion}

Acinetobacters have been considered members of a single species, A. calcoaceticus, with two biovars. However, new molecular methods of identification (DNA-DNA hybridisation) have divided the genus Acinetobacter into 19 DNA-DNA homologous groups $[9,20-22]$. The confusion that the new taxonomy has caused in the clinical laboratory is compounded by the fact that identification of the various species and DNA groups by phenotypic characteristics, many of which are assimilation of numerous carbon sources, is very difficult. The tests for identification of Acinetobacter spp. selected by Bouvet and colleagues [20,22] seem to be appropriate, but the large panel of phenotypic tests is tedious and time-consuming to perform. Simple identification schemes such as those of Gerner-Smidt et al. [12] and Tjernberg [13], along with antimicrobial susceptibility testing, may be useful for laboratories with limited resources and can be adapted for typing isolates, thus avoiding the need for expensive molecular methodology.

The increasing clinical importance of Acinetobacter spp. is attributed mainly to their capacity to cause nosocomial infections, particularly outbreaks in ICUs. The most important nosocomial acinetobacters belong to the $A c b$-complex $[9,23]$. The present study also identified more strains belonging to the $A c b$-complex than other phenotypes. Repeated isolation of an organism from the blood with related clinical findings is a strong indication of infection [24]. This study reports six patients with infection and two cases of colonisation in the ICU. The remaining three cases had a single positive blood culture along with positive trachial aspirate. Sporadic infections with Acinetobacter spp. were punctuated with outbreaks lasting for a month at different times. Outbreaks encountered during the months of Oct. 1996, Sept. 1997 and Feb. 1998 were caused by multi-drug-resistant strains of the $A c b$ complex (phenotypes 1 and 2). The source of infection was the ICU environment, where the epidemic Acinetobacter strain $(A c b-1)$ was isolated over a prolonged period. The $A c b-1$ phenotype strain from the environment (humidifier) was phenotypically indistinguishable from strains isolated from patients 8 and 10 , and was distinct from all other phenotypes. The 
Table 5. MIC values of three antibiotics against 28 Acinetobacter isolates

\begin{tabular}{|c|c|c|c|c|c|c|c|}
\hline \multirow[b]{2}{*}{ Antibiotic } & \multirow[b]{2}{*}{ Phenotype } & \multicolumn{5}{|c|}{ MIC (mg/L) } & \multirow[b]{2}{*}{$\begin{array}{c}\text { Resistance } \\
(\%)\end{array}$} \\
\hline & & $\begin{array}{l}\text { Number of } \\
\text { isolates }\end{array}$ & Range & MIC50 & MIC90 & $\begin{array}{c}\text { Geometric } \\
\text { mean }\end{array}$ & \\
\hline \multirow[t]{2}{*}{ Ceftazidime } & $A c b$-complex $(1-6)$ & 21 & $2-128$ & 16 & 32 & 14.98 & 43 \\
\hline & Other phenotypes*' & 7 & $1-4$ & 2 & 4 & 2 & 0 \\
\hline \multirow[t]{2}{*}{ Amikacin } & $A c b$-complex $(1-6)$ & 21 & $1->256$ & 32 & 256 & 23 & 48 \\
\hline & Other phenotypes & 7 & $0.5-128$ & 1 & 128 & 3.28 & 28.6 \\
\hline \multirow[t]{2}{*}{ Cefotaxime } & $A c b$-complex $(1-6)$ & 21 & $8-128$ & 32 & 64 & 29.96 & 43 \\
\hline & Other phenotypes & 7 & $0.5-8$ & 2 & 8 & 2.21 & 0 \\
\hline
\end{tabular}

*Includes phenotype nos. 7, 8, 9, 10, 11 and 12 .

inability to trace the source of the $A c b$ phenotype 2 strain, which was responsible for most of the infection occurring during Oct. 1996 was probably due to inadequate environmental sampling. The outbreak strain $A c b-1$ was distinguished from other $A c b$ phenotypes by the inability of the latter to utilise malonate. Although both outbreak phenotypes 1 and 2 of $A c b$-complex showed resistance to almost all the antibiotics tested by both methods, strains of $A c b-1$ phenotype were distinct because of their susceptibility to amikacin and netilmicin, unlike strains of $A c b$ phenotype-2. The simplified identification scheme for the assimilation tests showed similarity between DNA groups 1, 2, 3 and 13 and failed to speciate exactly within the $A c b$-complex. This is understandable because they are closely related genotypically [12]. Despite this inadequacy, phenotypic identification was possible for two species within the genus [20]. One strain was $A$. haemolyticus, isolated from an 86-yearold male patient suffering from recurrent bronchiectasis, the other was $A$. lwoffii, responsible for septicaemia in a 5-year-old female patient. Both $A$. haemolyticus and $A$. lwoffii are known to cause systemic infections $[25,26]$. Four isolates were closely related to phenotype $A$. lwoffii and one resembled the typical $A$. haemolyticus phenotype. Of four $A$. lwoffii-like organisms, one was isolated from a humidifier and indicated colonisation in ventilators in the ICU. The other three were responsible for systemic infections in elderly debilitated patients.

Traditional typing methods like phenotyping and antibiogram typing have an advantage over genotyping as they are readily available in all clinical microbiology laboratories. The biotyping procedure of Bouvet and Grimont is definitive [20]. However, genotyping methods such as ribotyping, pulse-field gel electrophoresis and PCR $[6,15,27,28]$ have a definitive advantage over phenotyping, as they clearly distinguish isolates within the $A c b$-complex. In the present study, all reference strains of DNA groups 1, 2, 3 and 13 showed almost identical phenotypic results. Laboratories with limited resources can still rely on a combination of data from two of the above-mentioned traditional approaches to typing. New molecular techniques have shown the genetic diversity within the $A c b$-complex to be greater than hitherto perceived [29]. Consideration should be given to placing the whole $A c b$-complex in a single species, $A$. calcoaceticus, which has priority over A. baumannii, and biotyping them further only for epidemiological purposes. As a large panel of assimilation tests is cumbersome, the simplified scheme followed in the present study is useful, as the discriminatory power is almost the same for both the methods [12].

The strains of $A c b$-complex could be clearly distinguished from non- $A c b$ phenotypes by their resistance to antimicrobial agents [9]. However, within the $A c b$ complex phenotypes 1 and 2 were more resistant than other $A c b$ phenotypes, as the other phenotypes were susceptible to three or more antibiotics tested and there is no major increase in the overall percentage of resistance in $A c b$ group. Variable resistance patterns like this have been observed by other workers [5], showing that not all phenotypes of $A c b$ are multi-drugresistant.

The disk diffusion technique showed that members of the $A c b$-complex were resistant to almost all antibiotics except for newer aminoglycosides and cephalosporins, i.e., amikacin, netilmicin and ceftazidime. Six strains of the $A c b$-complex (all $A c b$-2) were resistant to netilmicin, the sole antibiotic that was effective against all isolates of other phenotypes. With the agar dilution method, 14 strains had an MIC $\geqslant 256 \mathrm{mg} / \mathrm{L}$ for amikacin. This high-level resistance is probably due to extensive use of this antibiotic in the ICU. Ceftazidime was one of the second-line antibiotics used in this hospital to treat infections caused by multidrug-resistant gram-negative bacteria. Ceftazidime was also the most efficacious drug in the present study, as only $27 \%$ of strains were resistant, which can be attributed to its judicious use. In concordance with earlier studies $[5,6]$, the present study also encountered strains of the $A c b$-complex showing resistance to all drugs tested, which is of concern as infections caused by these organisms pose a therapeutic problem. It is surprising that the level of resistance to cefotaxime was moderate even though it was the commonest antibiotic used in the hospital. One environmental isolate $(A c b-1)$ was from the hands of an attending clinician (in the ICU) and was phenotypically indistinguishable from the outbreak strain and moderately resistant, suggesting 
colonisation by the $A c b$-complex strain and a source of infection for the patients.

Simple identification schemes and antimicrobial susceptibility testing provide a cost-effective approach to typing Acinetobacter spp. Although the above systems have certain limitations when compared to molecular methodologies, the distinction between resistant and susceptible acinetobacters at least, is useful for the effective clinical management of the infections caused by this group of organisms.

We thank Dr P. Gerner-Smidt (Statens Serum Institut, Copenhagen, Denmark) for generously providing Acinetobacter reference strains and the faculty of the Department of Microbiology (JIPMER, India) for their assistance and suggestions in the preparation of the manuscript.

\section{References}

1. Bergogne-Bérézin E, Joly-Guillou ML, Vieu JF. Epidemiology of nosocomial infections due to Acinetobacter calcoaceticus J Hosp Infect 1987; 10: 105-113.

2. Gerner-Smidt P. Endemic occurrence of Acinetobacter calcoaceticus biovar anitratus in an intensive care unit. $J$ Hosp Infect 1987; 10: 265-272.

3. Horrevorts A, Bergman K, Kollée L, Tjernberg I, Dijkshoorn L. Clinical and epidemiological investigations of Acinetobacter genospecies 3 in a neonatal intensive care unit. J Clin Micrbiol 1995; 33: $1567-1572$.

4. Tilley PAG, Roberts FJ. Bacteraemia with Acinebacter species: risk factors and prognosis in different clinical settings. Clin Infect Dis 1994; 18: 896-900.

5. Bello H, Gonzalez G, Dominguez M, Zemelman R, Garcia A Mella S. Activity of selected beta-lactams, ciprofloxacin, and amikacin against different Acinetobacter baumannii biotypes from Chilean hospitals. Diagn Microbiol Infect Dis 1997; 28: $183-186$.

6. Tankovic J, Legrand P, De Gatines G, Chemineau V, BrunBuisson C, Dural J. Characterization of a hospital outbreak of imipenem-resistant Acinetobacter baumannii by phenotypic and genotypic typing methods. J Clin Microbiol 1994; 32: 2677-2681.

7. Washington JA. An international multicenter study of blood culture practices. The International Collaborative Blood Culture Study Group. Eur J Clin Microbiol Infect Dis 1992; 11: 1115-1128.

8. Achar KN, Johny M, Achar MN, Menon NK. Communityacquired bacteraemic Acinetobacter pneumonia with survival. Postgrad Med J 1993; 69: 936-937.

9. Bergogne-Bérézin E, Towner KJ. Acinetobacter spp. as nosocomial pathogens: microbiological, clinical, and epidemiological features. Clin Microbiol Rev 1996; 9: 148-165.

10. De A, Deodhar L. Antibiotic resistance pattern and R-plasmids of Acinetobacter calcoaceticus subsp. antiratus. Indian $J$ Pathol Microbiol 1995; 38: 185-188.

11. Chopade BA, Patwardhan RB, Dhakephalkar PK. Acinetobacter infections in India: genetic and molecular biological studies and some approaches to the problem. In: Tropical diseases. Delhi, CSIR. 1994: 704-717.

12. Gerner-Smidt P, Tjernberg I, Ursing J. Reliability of phenotypic tests for identification of Acinetobacter species. $J$ Clin Microbiol 1991; 29: 277-282.

13. Tjernberg I. Antimicrobial susceptibility of Acinetobacter strains identified by DNA-DNA hybridisation. APMIS 1990; 98: $320-326$.

14. Gerner-Smidt P, Frederiksen W. Acinetobacter in Denmark: I Taxonomy, antibiotic susceptibility, and pathogenicity of 112 clinical strains. APMIS 1993; 101: 815-825.

15. Seifert H, Dijkshoorn L, Gerner-Smidt P, Pelzer N, Tjernberg I, Vaneechoutte M. Distribution of Acinetobacter species on human skin: comparison of phenotypic and genotypic identification methods. J Clin Microbiol 1997; 32: 2819-2825.

16. Barrow GI, Feltham RKA. Bacterial characters and characterization. In: Barrow GI, Feltham RKA (eds) Cowan and Steel's manual for the identification of medical bacteria, 3rd edn. Cambridge, Cambridge University Press. 1993: 1-45.

17. Stainer RY, Palleroni NJ, Doudoroff M. The aerobic pseudomondads: a taxonomic study. J Gen Microbiol 1966; 43: $159-271$.

18. Bauer AW, Kirby WMM, Sherris JC, Turck M. Antibiotic susceptibility testing by a standardized single disc method. $\mathrm{Am}$ $J$ Clin Pathol 1966; 45: 493-496.

19. National Committee for Clinical Laboratory Standards. Methods for dilution antimicrobial susceptibility tests for bacteria that grow aerobically. Approved standards M7-A4. Wayne, PA, National Committee for Clinical Laboratory Standards. 1997.

20. Bouvert PJM, Grimont PAD. Taxonomy of the genus Acinetobacter with the recognition of Acinetobacter baumannii sp nov., Acinetobacter haemolyticus sp. nov., Acinetobacter johnsonii sp. nov., and Acinetobacter junii sp. nov. and emended description of Acinetobacter calcoaceticus and Acinetobacter lwoffii. Int J Syst Bacteriol 1986; 36: 228-240.

21. Tjernberg I, Ursing J. Clinical strains of Acinetobacter classified by DNA-DNA hybridization. APMIS 1989; 97: 595-605.

22. Bouvet PJM, Jeanjean S. Delineation of new proteolytic species in the genus Acinetobacter. Res Microbiol 1989; 140: 291-299.

23. Gerner-Smidt P. Taxonomy and epidemiology of Acinetobacter infections. Rev Med Microbiol 1995; 6: 186-195.

24. Garner JS, Jarvis WR, Emori TG, Horan TC, Hughes JM. CDC definitions for nosocomial infections, 1988. Am J Infect Control 1988; 16: 128-140.

25. Castellanos Martínez E, Telenti Asensio M, Rodrígues Blanco VM, Rodgriguez Suárez ML, Morena Torrico A, Cortina Llosa A. Infective endocarditis of an interventricular patch caused by Acinetobacter haemolyticus. Infection 1995; 23: 243-245.

26. Seifert H, Strate A, Schulze A, Pulverer G. Bacteremia due to Acinetobacter species other than Acinetobacter baumannii. Infection 1994; 22: 379-385.

27. Gouby A, Carles-Nurit MJ, Bouziges N, Bourg G, Mesnard R, Bouvet PJM. Use of pulsed-field gel electrophoresis for investigation of hospital outbreaks of Acinetobacter baumannii. $J$ Clin Microbiol 1992; 30: 1588-1591.

28. Webster CA, Crowe M, Humphreys H, Towner KJ. Surveillance of an adult intensive care unit for long-term persistence of a multi-resistant strain of Acinetobacter baumannii. Eur $J$ Clin Microbiol Infect Dis 1998; 17: 171-176.

29. Gerner-Smidt P, Tjernberg I. Acinetobacter in Denmark: II. Molecular studies of the Acinetobacter calcoaceticus-Acinetobacter baumannii complex. APMIS 1993; 101: 826-832. 